\section{Ärztliche Verschreibung von Heroin \\ an chronische, therapieresistente Methadonpatienten in den Niederlanden} Niederlanden

Zusammenfassung: Dieser Artikel beginnt mit einer kurzen epidemiologischen Beschreibung der Heroinabhängigkeit in den Niederlanden und einem Überblick über das gegenwärtige Behandlungssystem unter besonderer Berücksichtigung der oralen Substitution mit Methadon. Es folgt ein kurzer historischer Abriss der Diskussion um die Verschreibung von Heroin an abhängige Patienten. Dabei zeigt es sich, dass Alternativen zum Methadon in den Niederlanden seit den frühen 80er Jahren als wesentlich angesehen wurden. Frühere Versuche mit intravenösem Morphin, intravenösem Methadon und oralem Dextromoramid (Palfium ${ }^{\circledR}$ ) waren nicht so erfolgreich wie erhofft und wurden nicht in die tägliche Praxis aufgenommen. Schließlich erleichterten die jüngsten Erfahrungen mit der ärztlichen Verschreibung von Heroin in der Schweiz die Entscheidung, in den Niederlanden eine wissenschaftliche Studie über die ärztliche Verschreibung von Heroin in die Wege zu leiten. Dieser Artikel gibt eine detaillierte Beschreibung der randomisierten klinischen Studie, die derzeit in den Niederlanden durchgeführt wird und in der die Wirksamkeit von zusätzlich verschriebenem Heroin untersucht wird. Am Ende folgt eine kurze Diskussion der verschiedenen Studienergebnisse über mögliche Konsequenzen für zukünftige Behandlungsoptionen.

Schlüsselwörter: Heroinabhängigkeit - Behandlungseffektivität - Heroinbehandlung

Medical Prescribing of Heroin for Chronic, Therapy Resistant Methadone Patients in the Netherlands: This paper begins with a brief description of the epidemiology of heroin addiction in the Netherlands and an overview of the current treatment system, with special emphasis on oral methadone maintenance. It is followed by a short history of the debate over the prescription of heroin to addicted patients. This history reveals that alternatives to methadone have been viewed as essential in the Netherlands since the early 1980s. Earlier experiments with intravenous morphine, intravenous methadone and oral dextromoramide (Palfium ${ }^{R}$ ) were not as successful as had been hoped and precluded their introduction in daily practice. Finally, recent experiences in Switzerland with the medical prescription of heroin facilitated the decision to initiate a scientific study of the medical co-prescription of heroin in the Netherlands. The paper provides a detailed description of the randomized clinical trial regarding the effectiveness of co-prescribed heroin that is now under-

Suchttherapie 2000; $1: 71-82$

(c) Georg Thieme Verlag Stuttgart · New York

ISSN 1439-9903
Wim van den Brink ${ }^{1,2}$, V. M. Hendriks',3, J. M. van Ree',4

1 Zentrales Komitee für Behandlung von Heroinabhängigen (CCBH), Utrecht

2 Institut für Suchtforschung der Universität Amsterdam

3 Zentrales Komitee für Heroinabhängige; Forschungszentrum des Instituts für psychische Gesundheit Parnassia, Den Haag

${ }^{4}$ Rudolf-Magnus-Institut für Neurowissenschaften der Universität Utrecht

way in the Netherlands. It concludes with a brief discussion of the likely consequences for future treatment options of different study outcomes.

Key words: Heroin-Dependence - Treatment Effectiveness Heroin Maintenance Treatment

\section{Einführung}

Im Herbst 1972 gelangte Heroin in die Niederlande. In den ersten Jahren beschränkte sich sein Gebrauch weitgehend auf die Population niederländischer Abstammung und es wurde in erster Linie intravenös konsumiert. 1975 kam es infolge der Unabhängigkeit Surinams, einer früheren niederländischen Kolonie im nördlichen Südamerika, zu einem raschen Anstieg der Zahl der Heroinkonsumenten. Zu dem Zeitpunkt emigrierte nahezu die Hälfte der surinamischen Bevölkerung in die Niederlande und ließ sich überwiegend in Amsterdam, Rotterdam, Den Haag und einigen anderen städtischen Regionen nieder. Junge surinamische Männer übernahmen schließlich eine führende Rolle beim Straßenhandel mit Heroin und viele von ihnen wurden selbst zu Konsumenten. Dabei hielten sie jedoch an ihrer eigenen Art der Drogenverabreichung fest, d.h. sie inhalierten statt zu injizieren [1]. Seit damals ist die geschätzte Anzahl der Heroinkonsumenten von $10000 \mathrm{im}$ Jahre 1977 auf 20000 (1977) und 30000 (1983) gestiegen [2]. Es scheint, dass sich die Gesamtzahl der Heroinabhängigen in den Niederlanden seit 1984 weitgehend stabilisiert hat, wobei es in den letzten Jahren wahrscheinlich zu einem leichten Rückgang kam [3,4]. Die Gesamtzahl der Heroinabhängigen in den Niederlanden wird gegenwärtig auf 25000 geschätzt [2].

Die allgemeine Situation ist geprägt von einer relativ stabilen Population von Heroinabhängigen bei einer niedrigen Inzidenz für neue Fälle und einer niedrigen Sterberate. Dies zeigt sich auch in dem Umstand, dass das durchschnittliche Alter der Population der Methadonsubstituierten in Amsterdam seit 1984 jedes Jahr um ungefähr 10 Monate zugenommen hat (1984 betrug das Durchschnittsalter 28,2 Jahre; 1997 lag das Durchschnittsalter bei 38,8 Jahren). Außerdem fiel die Prozentzahl der Heroinabhängigen unter 26 Jahre, die sich in einer Methadon-Substitutionstherapie befanden, von $28 \% \mathrm{im}$ Jahre 1985 auf 3\% im Jahr 1997 [5]. Ein ähnliches Muster ergab sich in Rotterdam, wo 1988 28\% der Patienten in einer Methadon-Substitutionstherapie unter 25 Jahre alt waren, während es 1995 nur noch 13\% waren, wobei 1988 das 
Durchschnittsalter bei 29,3 Jahren und 1995 bei 34,2 Jahren lag [6]. Zur gleichen Zeit änderte sich auch das Verhältnis von intravenös Konsumierenden zu Heroinrauchern: Es nahm der Anteil der Abhängigen zu, die hauptsächlich Heroin gebrauchen, indem sie die zuvor auf einer Aluminiumfolie erhitzten Dämpfe der Heroinbase inhalieren („chasing the dragon“).

Diese stabile, alternde Population von Heroinabhängigen wird von einem umfassenden Behandlungssystem versorgt, dessen Leistungen kostenlos sind und in dem es keine oder nur kurze Wartelisten gibt. Dieses System umfasst abstinenzorientierte Behandlungsmöglichkeiten (z.B. stationäre und ambulante Entgiftung, Methadonentgiftung, stationäre Therapien und therapeutische Wohngemeinschaften), stellt aber auch eine große Bandbreite an Einrichtungen zur Verfügung, die eher auf eine Stabilisierung und Schadensreduzierung abzielen als auf Abstinenz (z.B. Methadon-Substitutionsbehandlung, Nadel- und Spritzentausch, Arbeitsprojekte, Unterbringungsstätten und Konsumräume). Es wird geschätzt, dass je nach den örtlichen Gegebenheiten 65-85\% aller Heroinabhängigen gegenwärtig in irgendeiner Art von Kontakt mit dem Behandlungssystem stehen $[2,7]$.

Häufig beginnen die Patienten die Behandlung in der Hoffnung, abstinent zu werden. Im Verlauf ihrer Drogen- und Behandlungskarrieren wechseln jedoch viele derjenigen, die ihre Sucht nicht aufgeben, von einer Abstinenzbehandlung zu einer Form der Schadensminimierung. Gewöhnlich geschieht dies über die Teilnahme an einem Methadon-Substitutionsprogramm, sei es mit oder ohne zusätzliche Anwendung von anderen pharmakologischen Therapien (z.B. Dreifachtherapie bei AIDS, Antidepressiva bei einer komorbiden affektiven Störung) und psychotherapeutischen Interventionen (z.B. Beratung, Fertigkeitentraining) oder psychosozialen Interventionen (begleitende Wohngruppen, Arbeitsprojekte).

Bei der ärztlichen Heroinverschreibung handelt es sich um eine letzte Behandlungsoption, die nur für chronisch Heroinabhängige vorgesehen ist, die wiederholt erfolglos an anderen verfügbaren Behandlungsformen teilgenommen haben, einschließlich einer Behandlung entsprechend dem aktuellen Forschungsstand in einem Methadon-Substitutionsprogramm. Es ist darum sehr wichtig, eine angemessene Vorstellung von der Methadonbehandlung in den Niederlanden und den Ergebnissen, die damit erzielt worden sind, zu haben.

\section{Die Behandlung mit Methadon in den Niederlanden}

Die Verschreibung von Methadon als Behandlungsmethode wurde in den Niederlanden 1968 begonnen. Während der ersten Jahre wurde Methadon an morphinabhängige Patienten verschrieben. Nach der Einführung von Heroin in den Niederlanden 1972 waren die Behandlungen mit Methadon in erster Linie darauf ausgerichtet, Abstinenz von der Heroinabhängigkeit zu erreichen. Im Allgemeinen litten diese Methadon-Reduzierungsprogramme unter hohen Abbrecherraten und dabei bestand ein ernsthaftes Risiko, den Kontakt zu vielen Abhängigen zu verlieren. Parallel zum raschen Anstieg der Anzahl von Heroinabhängigen während der 70er und zum Auftauchen von HIV/AIDS Mitte der 80er Jahre verlagerte sich das Ziel der Methadonverschreibung vom Erreichen der Abstinenz hin zu Stabilisierung und Schadensminimierung. Die vorrangigen Ziele der Methadon-Substitutionsprogramme bestanden nun bei denjenigen Patienten, die eine Beratung ablehnten und die Einnahme illegaler Drogen fortsetzten, in der Verhütung riskanten Verhaltens und der Bereitstellung ärztlicher Betreuung, was durch regelmäßigen Kontakt zu den Abhängigen erreicht werden sollte. In diesem Zeitraum, in dem ein erhöhtes Infektionsrisiko und eine gesteigerte Notwendigkeit bestand, mit dem Abhängigen wenigstens in Kontakt zu bleiben (im Hinblick auf Präventivmaßnahmen gegen AIDS), wurden die hohen Abbrecherraten in den Methadonprogrammen sowohl von den Therapieeinrichtungen als auch den Politikern als inakzeptabel angesehen.

Gegenwärtig sind viele der niederländischen Methadon-Substitutionsprogramme niedrigschwellig angelegt. Sie sind gekennzeichnet durch das Fehlen einer obligatorischen Beratung; es werden keine Sanktionen vorgenommen, wenn durch Urinkontrollen der Gebrauch illegaler Substanzen nachgewiesen wird, und es werden relativ niedrige Methadondosierungen gegeben. Diese Programme zielen darauf ab, die Gesundheitsrisiken zu reduzieren und die Lebensqualität der Abhängigen zu verbessern, die nicht bereit sind, ihren Drogenkonsum aufzugeben. Aufgrund ihres niedrigschwelligen Charakters wird mit Hilfe dieser Programme in den Niederlanden ein hoher Prozentsatz der Heroinabhängigen erreicht (50-80\%), einschließlich derjenigen, die bei ihren früheren Entwöhnungsbemühungen keinen Erfolg hatten [8]. Im Gegensatz dazu sind in den USA die meisten Methadonprogramme „hochschwellig“ angelegt und richten sich an die Abhängigen, die imstande sind, eine entsprechende „Leistung“ zu erbringen; das Ziel dieser Programme besteht in dem Erreichen von Abstinenz, wobei lediglich 15-20\% der Heroin konsumierenden Population erreicht wird [9]. Aufgrund der Unterschiede zwischen den niederländischen und US-amerikanischen Methadonversuchen sowie der Populationen, die diese in Anspruch nehmen, können die Ergebnisse der amerikanischen Methadonuntersuchungen nicht einfach auf die niederländische Situation übertragen werden.

In den Niederlanden sind zwar keine kontrollierten Studien über die Wirksamkeit von Methadonbehandlungen durchgeführt worden, es liegen jedoch umfangreiche klinische Erfahrungen mit der Verabreichung von Methadon vor und Followup-Studien gewähren einen Einblick in deren Ergebnisse. 1988 wurde in den Niederlanden eine Untersuchung über die Gabe von Methadon durchgeführt [10]. Nach dieser Studie belief sich die geschätzte Zahl der Heroinabhängigen in den Niederlanden zu diesem Zeitpunkt auf 24000. Davon standen schätzungsweise 17000 Abhängige (71\% der Gesamtzahl) wenigstens einmal im Verlauf des vorangegangenen Jahres in Kontakt mit einem Behandlungsprogramm (Abb.1). Von dieser Gruppe befanden sich ca. 4500 in einer Abstinenztherapie und ungefähr 12500 erhielten regelmäßig Methadon, die meisten von ihnen über mehrere Jahre hinweg (die durchschnittliche Teilnahmezeit im Programm lag bei annähernd 8 Jahren). Nach Einschätzung der Teams, die diese MethadonSubstitutionsprogramme betreuten, waren 36\% der $12500 \mathrm{~Pa}-$ tienten gut eingestellt, das heißt, dass sie wenig oder gar keinen Nebenkonsum illegaler Drogen zeigten, mit den Behandlungsbemühungen einverstanden waren und ein gewisses $\mathrm{Maß}$ an sozialer Integration aufwiesen. Weitere $40 \%$ waren nicht angemessen eingestellt, das heißt, dass sie häufig illegale Drogen einnahmen, keine gute Compliance bezüglich der Behandlungsbemühungen zeigten und nur ein geringes Maß 


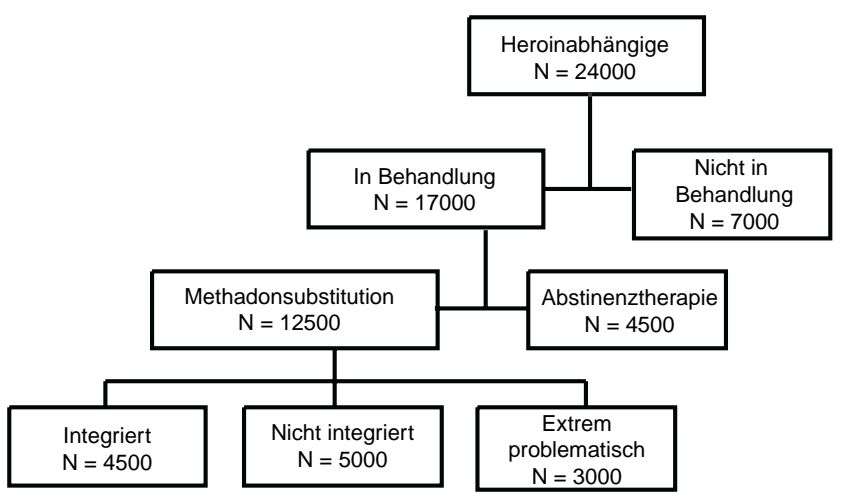

Abb.1 Die Situation der Drogenbehandlung in den Niederlanden.

an sozialer Integration erreichten. 24\% (3000 Personen) erwiesen sich schließlich als extrem problematisch. Sie nahmen täglich verschiedene illegale Substanzen ein, ließen Symptome erkennen, die auf physische und/oder psychische Probleme hinwiesen, zeigten hohe Raten von Kriminalität und waren in keiner Weise sozial integriert.

Die Daten von 1991 lassen erkennen, dass drei Viertel (78\%) aller methadonsubstituierten Patienten weiterhin Heroin nahmen und dass mehr als ein Drittel (37\%) täglich Heroin konsumierte [10]. Zu dieser Zeit boten nahezu alle MethadonSubstitutionsprogramme neben der Substitutionsdroge auch ein breites Spektrum von Leistungen an, die psychosoziale Beratung, ärztliche Beratung und Behandlung, Sozialarbeit und - in einigen Einrichtungen - Psychotherapie beinhalteten. Trotz dieses vorhandenen Angebotes nahmen nur 49\% der Patienten Sozialarbeit, 28\% ärztliche Beratung, 13\% psychiatrische Beratung, 2\% Psychotherapie, 2\% Familientherapie und 6\% Gruppentherapie in Anspruch [10].

Diese älteren Daten werden von einer neueren Studie aus Amsterdam unterstützt [4]. Diese Untersuchung zeigte, dass 5545 Heroinabhängige, die in Amsterdam lebten, zwischen 1994 und 1995 Methadon erhalten hatten. Basierend auf den Ergebnissen von Urinkontrollen fand man heraus, dass 65$70 \%$ von ihnen weiterhin regelmäßig oder sogar täglich Heroin nahmen. Diese hohe Rate von illegalem Heroinkonsum kann jedoch nicht - wie dies manchmal geschieht - mit dem Verweis auf niedrige Dosierungen (weniger als $50 \mathrm{mg}$ ) des verschriebenen Methadon erklärt werden, da in Amsterdam 1995 die durchschnittliche tägliche Methadondosis fast $60 \mathrm{mg}$ betrug [4].

Zusätzlich zu den 12500 Patienten in Methadon-Substitutionsprogrammen gab es in den Niederlanden 1995 mehr als 1400 Aufnahmen in allgemeine psychiatrische Krankenhäuser und mehr als 3000 Aufnahmen in Suchtbehandlungskliniken, die in Zusammenhang mit Drogen standen [7]. Schließlich bietet auch das niederländische Beratungszentrum für $\mathrm{Al}$ kohol und Drogen ambulante drogenfreie Therapien an. In diesem Zusammenhang ist es wichtig zu beachten, dass es nicht möglich ist, diese Zahlen einfach zu addieren, um zu einer Ziffer für das Land insgesamt zu kommen, da einige Patienten während des Registrierungszeitraums mehr als eine der oben erwähnten Einrichtungen in Anspruch nahmen und daher mehr als einmal erfasst sein könnten. Es ist jedoch sehr wahrscheinlich, dass Umfang und Art der Leistungen, die den Heroinabhängigen gegenwärtig geboten werden, denen von 1988 sehr ähneln [10].

1993 untersuchte das Niederländische Institut für Psychische Gesundheit (NcGv) 1900 aktuelle und ehemalige Patienten des Beratungszentrums für Alkohol und Drogen [11]. Die Ergebnisse dieser Untersuchung zeigten, dass 62\% der Methadonpatienten mit der erhaltenen Behandlung sehr zufrieden waren. Fast $60 \%$ von ihnen berichteten von einer Reduzierung ihres Heroinkonsums aufgrund dieser Behandlung und nahezu ein Drittel bezeichnete sich als abstinent, was den Gebrauch illegaler Drogen betraf. Trotz dieser positiven Resultate sagten $47 \%$ der Patienten, dass es wegen des Methadons schwieriger für sie gewesen sei, abstinent zu werden, und $10 \%$ berichteten, die Methadonbehandlung hätte keinerlei positiven Einfluss auf sie gehabt. Die erwähnten Zahlen sind denen sehr ähnlich, die Driessen [10] zu einem früheren Zeitpunkt zusammengetragen hatte. Damals hatten $47 \%$ eine oder mehrere schwer wiegende Klagen geäußert, insbesondere über die Ausgabezeiten, den Mangel an Take-homeDosen und den unerwünschten Kontakt mit anderen Drogenabhängigen.

Es werden in den Niederlanden mehrere unterschiedliche Behandlungssettings in den Methadon-Programmen angeboten. Es stehen jedoch keine Daten zur Verfügung über den Zusammenhang zwischen der Methadondosis, den verschiedenen in den Programmen angebotenen Beratungsmöglichkeiten und den Behandlungsergebnissen. Die vom Städtischen Gesundheitsdienst von Amsterdam erhobenen Zahlen zeigen jedoch, dass es $z u$ einem Anstieg der durchschnittlichen täglichen Methadondosis gekommen ist: von $43 \mathrm{mg}$ im Jahre 1989 auf $56 \mathrm{mg}$ im Jahre 1995 [4]. Aus anderen Städten der Niederlande wurde über eine ähnliche Entwicklung berichtet. Diese Durchschnittsmenge liegt jedoch immer noch unter der empfohlenen Dosierung, die in den Programmen in den USA verwendet wird [12]. Nach den amerikanischen Richtlinien sollte die tägliche Effektivdosis ca. $80 \mathrm{mg}$ (plus/minus $20 \mathrm{mg}$ ) betragen. Eine landesweite Untersuchung in den USA hat jedoch gezeigt, dass die Dosierungsgrenzen vieler Programme unterhalb dieser Wirkbereiche liegen und dass es in vielen Programmen eine zeitliche Begrenzung für die maximale Dauer der erlaubten Teilnahme an diesen Programmen gibt [13]. Angesichts der Tatsache, dass die Mehrzahl der Patienten, die in den Niederlanden Methadon bekommen, eher nicht imstande oder willens sind, ihren Heroinkonsum einzustellen, wären höhere Methadondosierungen, die verhindern würden, dass der Patient die euphorisierende Wirkung des Heroins erleben könnte [14], für den Betreffenden oft nicht zu akzeptieren. Infolgedessen würde das Verschreiben höherer Methadondosierungen eher die Rate der Therapieabbrecher ansteigen lassen und auf diese Weise eines der vorrangigen Ziele des niederländischen Methadon-Behandlungssystems untergraben: aus Gründen der Prävention, der ärztlichen Beobachtung und Behandlung so viele Abhängige wie möglich zu erreichen.

Die Wirksamkeit dieses pragmatischen Ansatzes wird unter anderem auch durch die relativ geringe Anzahl von intravenös Drogenkonsumierenden mit AIDS in den Niederlanden $(12,4 \%)$ im Vergleich zu den USA (24\%) und Europa insgesamt (38\%) belegt [15]. Außerdem ist die Zahl der Todesfälle, die auf Drogengebrauch zurückzuführen sind (als primäre oder 
sekundäre Todesursache), in den Niederlanden recht niedrig: 1995 wurden lediglich 33 Todesfälle niederländischer Einwohner registriert, die in Zusammenhang mit Drogen standen [16]. In Amsterdam und Rotterdam - die Städte, die mit Abstand die höchste Zahl an Heroinkonsumenten und -abhängigen aufweisen - gab es 1996 in erstgenannter Stadt 16 und in letztgenannter Stadt 14 Tote [16].

Insgesamt deuten diese Ergebnisse darauf hin, dass eine Teilnahme an einem Methadon-Substitutionsprogramm in den Niederlanden fast überall möglich ist. Wenigstens 50\% aller Heroinabhängigen nehmen derzeit an einem der Programme teil, das vorgesehen ist für die stabile und älter werdende Population chronischer Heroinabhängiger mit langer Sucht- und Behandlungskarriere. Es konnte ebenfalls festgestellt werden, dass eine erhebliche Anzahl von Methadonpatienten regelmäßig Heroin und andere illegale Drogen konsumiert. Schätzungsweise 8000 der 12500 Methadonpatienten bleiben in ihren gesundheitlichen und psychosozialen Funktionen unterhalb des Optimalbereichs. Ungefähr 3000 von ihnen zeigen ein in hohem Grad kriminelles Verhalten. In zunehmendem Maß ist man sich dieser enttäuschenden Ergebnisse bewusst geworden, zu denen es trotz der intensiven professionellen Bemühungen, die Lebensbedingungen dieser chronisch abhängigen Patienten zu verbessern, gekommen ist. Wiederholt wurde gefordert, die durch das kriminelle Verhalten dieser Gruppe entstehenden öffentlichen Probleme zu reduzieren. Aufgrund dieser Schwierigkeiten wurden verschiedene Versuche unternommen, diese chronischen therapieresistenten Heroinabhängigen mit anderen Opioiden als dem oralen Methadon zu behandeln. Im Folgenden werden diese Bemühungen kurz diskutiert.

\section{Die Verschreibung von anderen Opioiden als oralem Methadon in den Niederlanden}

In den Niederlanden sind drei klein angelegte Versuche durchgeführt worden, in denen die Substitutionsbehandlung mit rezeptpflichtigen Opioiden anstatt mit oralem Methadon untersucht wurde. In den späten 70er Jahren begann der behandelnde Arzt einer niedrigschwelligen Tagesstätte für chronische Heroinabhängige in Amsterdam mit der Verschreibung von Morphin i.v. und Amphetaminen an fünf Patienten. 1983 veröffentlichte er im Maandblad Geestelijke Volksgezondheid die positiven Ergebnisse dieser Interventionen [17] und verhandelte mit dem Städtischen Gesundheitsdienst von Amsterdam (GG\&GD) über eine Erweiterung des Programms [18]. Nach einer hitzigen Diskussion und der Beendigung des Abgabeprogramms in der Tagesstätte genehmigte der Gesundheitsminister ein Programm zur Abgabe von Morphin an 37 Patienten. Zur gleichen Zeit kündigte der Stadtrat von Amsterdam Pläne zur Einleitung eines groß angelegten Heroinabgabeprogramms für ca. 300 Drogenkonsumenten an. Dieser Vorschlag wurde jedoch von der niederländischen Regierung zurückgewiesen und das Programm wurde niemals durchgeführt $[18,19]$.

Das Morphinabgabeprogramm wurde Ende 1983 mit einer Gruppe von 37 extrem problematischen Heroinabhängigen begonnen. Die Versuchsdauer war auf zwei Jahre angelegt. Die Mehrzahl der Patienten erhielt eine Kombination von Morphin i.v. zusammen mit einer Grunddosis Methadon oral. Die Ergebnisse dieser Studie legten eine positive Wirkung nahe, was sich an einer Abnahme des illegalen Heroinkonsums und niedrigeren Kriminalitätsraten bei annähernd der Hälfte der Teilnehmer zeigte. Diese Ergebnisse konnten jedoch nicht einfach der Verschreibung von Morphin i.v. zugeschrieben werden, da es in dieser Untersuchung keine Kontrollgruppe gab. Ein Schwachpunkt der Studie war auch der Therapieabbruch von elf Teilnehmern, welche die Behandlung innerhalb des ersten Jahres aufgaben, weil sie mit dem Morphin unzufrieden waren und/oder weil sie unter den histaminergen Reaktionen litten, die sich nach der Verabreichung einstellten [20, 21]. 1993 - zehn Jahre nach Beginn des Projektes, das eigentlich nur auf zwei Jahre angelegt war - erhielten sieben der ursprünglich 37 Patienten weiterhin Morphin i.v. Von den Übrigen waren vierzehn Patienten zu einer oralen Methadon-Substitutionstherapie zurückgekehrt, ein Patient war bekanntermaßen drogenfrei und fünfzehn waren verstorben (fünf hatten Suizid verübt, sechs waren an AIDS gestorben, zwei hatten Lungenkrebs bekommen und zwei waren an einer Überdosis Drogen gestorben). Diese Zahlen zeigen deutlich, wie alarmierend hoch die Sterberate ist. Bei der Mehrzahl der Todesfälle ist jedoch anzunehmen, dass sie nicht in Zusammenhang mit dem Programm stehen, sondern lediglich den Schweregrad des pathologischen Zustands der Patienten zum Zeitpunkt der Aufnahme in die Studie widerspiegeln. Das Versuchsprogramm hat gezeigt, dass die Verschreibung injizierbarer Opioide durchführbar ist, dass nur geringe Mengen Morphin auf den Schwarzmarkt gelangen und dass es bei einigen der Patienten zu einer Besserung kam. Dieser Versuch wurde nach Beendigung nicht mit neuen Patienten wiederholt, da die Mehrheit - wenn vor die Wahl gestellt - es vorzog, Methadon i.v. zu nehmen.

1990 wurde ein zweiter Quasi-Versuch gestartet, als der Städtische Gesundheitsdienst von Amsterdam begann, injizierbares Methadon an 30 unkontrollierbare und schwer abhängige AIDS-Patienten, die sich in einem äußerst schlechten Gesundheitszustand befanden, zu verschreiben. Für annähernd die Hälfte der Teilnehmer musste die Abgabe eingestellt werden, da sie sich nicht an die Behandlungsvorgaben hielten. In der verbleibenden Gruppe verstarben in der Folgezeit viele. Bei den Patienten, welche die Behandlung fortsetzten, zeigten sich deutliche Verbesserungen in ihrer therapeutischen Beziehung zu den Behandlungsteams. Des Weiteren ging der Heroingebrauch dieser Patienten deutlich zurück, obwohl er nicht vollständig eingestellt wurde. Den Patienten zufolge erzeugt Methadon i.v. ein Hochgefühl, das jedoch dem durch Heroin verursachten Gefühl nicht gleichzusetzen ist [4]. Angesichts dieser weit verbreiteten Überzeugung sollte es nicht überraschen, dass die überwältigende Mehrheit der niederländischen Methadonpatienten nicht daran interessiert ist, an einem Programm mit injizierbarem Methadon teilzunehmen [11].

Im Jahre 1995 startete der Städtische Gesundheitsdienst von Amsterdam ein drittes experimentelles Behandlungsprogramm. Bei diesem Versuch wurde zusätzlich zu oralem Methadon orales Dextromoramid (Palfium ${ }^{\circledR}$ ) verschrieben. Es nahmen 53 schwer abhängige, nicht i.v. Heroinkonsumenten teil, die im Durchschnitt seit 21 Jahren abhängig waren und die alle auf frühere Methadonbehandlungen nicht gut angesprochen hatten. Das Ziel dieser neuen Behandlungsstrategie bestand darin, die Leiden der Patienten zu mindern und ihre Drogenzufuhr zu stabilisieren. Bedauerlicherweise ist das 
Projekt nicht systematisch ausgewertet worden. Der allgemeine Eindruck des Behandlungsteams war jedoch, dass die Behandlung mit Palfium zu einer Verbesserung der sozialen Funktionen der Patienten und zu einer besseren Beziehung zu den therapeutischen Einrichtungen führte. Eine klinische Auswertung deutete darauf hin, dass bei den meisten Patienten der Heroingebrauch zurückging; ein Teilnehmer stellte den Konsum ganz ein. Bei den Urinanalysetests zeigte sich keine Zunahme im Kokaingebrauch. Die Beendigung der Verschreibungen bei den Patienten, für die sich kein Nutzen in der Palfiumbehandlung ergeben hatte, brachte keine Probleme mit sich [22].

Diese Versuche, den medizinischen und psychosozialen $\mathrm{Zu}$ stand chronischer, therapieresistenter Heroinabhängiger $\mathrm{zu}$ verbessern, sind Zeichen einer regen Diskussion über die ärztliche Verschreibung von anderen Opioiden als oralem Methadon. Jedoch richteten sich die experimentellen Behandlungsprogramme bisher an sehr kleine Gruppen von Heroinabhängigen mit eher speziellen Bedürfnissen und, wie bereits erwähnt wurde, sind die Ergebnisse dieser Versuche nicht ausreichend dokumentiert worden. Diese Einschränkungen lassen Raum für Zweifel an der Wirksamkeit der Behandlungen und daran, ob es möglich ist, diese Ergebnisse zu verallgemeinern. Angesichts dieser Zweifel griff das Komitee für Pharmakologische Interventionen bei Drogenabhängigkeit des Gesundheitsrates der Niederlande (1995) bei der Diskussion darüber, ob eine ärztliche Verschreibung von Heroin an niederländische Abhängige wünschenswert ist, auf die Auswertungsergebnisse der wissenschaftlichen Versuche mit Heroin in anderen Ländern zurück. Dabei handelte es sich um Programme aus Großbritannien [23] und der Schweiz [24]. In den nachfolgenden Abschnitten werden diese Ergebnisse zusammenfassend dargestellt.

\section{Die Wirksamkeit der Heroinverschreibung}

Im Laufe der letzten 40 Jahre haben verschiedene Länder Erfahrungen mit der Verschreibung von Heroin an Abhängige gesammelt. Begonnen wurde damit in den späten 50er und frühen 60er Jahren, als einzelne Ärzte in Großbritannien anfingen, Heroin an Abhängige zu verschreiben, um diese damit zu substituieren [25]. 1968 übernahmen die Drogenkliniken des NHS (National Health Service) die Verantwortung für das Verschreiben injizierbaren Heroins von den einzelnen Ärzten, denen es nun nicht länger erlaubt war, Drogen an Abhängige zu verschreiben. In den späten 60er und frühen 70er Jahren begannen diese Kliniken von der Verschreibung injizierbaren Heroins auf injizierbares Methadon umzustellen und seit Mitte der 70er Jahre von injizierbarem auf orales Methadon [26]. In diesem Zeitraum (1972-1975) wurde eine randomisierte klinische Studie durchgeführt, in der die Wirksamkeit der ärztlichen Verschreibung von injizierbarem Heroin (die alte Standardbehandlung) mit der ärztlichen Verschreibung von oralem Methadon (die neue Standardbehandlung) verglichen wurde [23]. Die untersuchte Population bestand aus 96 Patienten mit einem Durchschnittsalter von 23,9 Jahren (von 18 bis 32 Jahren), einer durchschnittlichen Abhängigkeitskarriere von 5,9 Jahren und einem täglichen i.v. Heroingebrauch von mindestens drei Monaten vor Beginn der Studie. Alle Teilnehmer waren fest entschlossen, ihren Drogenkonsum fortzusetzen. Die verschriebenen Heroindosen reichten von $30 \mathrm{mg}$ bis $120 \mathrm{mg}$ und die oralen Methadondosen von $10 \mathrm{mg}$ bis 120 mg täglich, wobei die Dosierungen meistens zwischen $40 \mathrm{mg}$ und $80 \mathrm{mg}$ täglich lagen.

Die Studie erbrachte insofern unklare Ergebnisse, als dass sich keine deutlichen Hinweise dafür fanden, dass eine der beiden Behandlungsformen bessere Ergebnisse zur Folge hatte als die andere. Beide Therapiemöglichkeiten zeigten positive Ergebnisse in einzelnen Bereichen und negative in anderen. So besuchten die Heroinpatienten beispielsweise die Klinik mit größerer Regelmäßigkeit als die Methadonpatienten. Bei Erstgenannten kam es auch zu einer größeren Reduzierung ihres illegalen Heroinkonsums sowie der kriminellen Aktivitäten. Bei keiner der beiden Gruppen konnten signifikante Änderungen beobachtet werden bezüglich der Arbeitsleistung, der Wohnsituation, der körperlichen Komplikationen oder der Ernährungsprobleme, die aus ihrem Drogengebrauch resultierten. Während die Mehrheit derjenigen, denen eine Heroinverschreibung verweigert wurde, fortfuhr, sich illegales Heroin zu spritzen, und die Klinik nur dann aufsuchte, wenn sie eine bestimmte Leistung benötigten, wurde ungefähr ein Fünftel erfolgreich auf orales Methadon eingestellt und ein weiteres Fünftel wurde abstinent [26]. Nach Meinung der Untersucher legen die Ergebnisse nahe, dass der Vorzug eines bestimmten Ansatzes von den festgelegten Prioritäten der verschiedenen Ergebnisbereiche abhängig ist. Es besteht die Wahl, entweder einer kleinen Zahl von Abhängigen zu helfen, mithilfe des oralen Methadons ihren Drogenkonsum zu beenden, oder man kann versuchen, mit injizierbarem Heroin so viele Abhängige wie möglich zu erreichen, um ihre Situation zu verbessern.

In dieser frühen Studie wurden die meisten Patienten der Heroinverschreibungsgruppe überzeugt, auf orales Methadon umzusteigen. Dies veranlasste die Untersucher zu der Vermutung, dass die Gabe einer Erhaltungsdosis von injizierbarem Heroin während eines begrenzten Zeitraumes nicht notwendigerweise zu irreversiblen Situationen mit potenziell ungünstigen Konsequenzen für den langfristigen Gesundheitszustand der Patienten führen muss [23]. Die Autoren wiesen darauf hin, dass die Ergebnisse keine eindeutigen Hinweise dafür lieferten, dass eine der beiden Behandlungsformen der anderen überlegen wäre. Trotz dieses Vorbehalts wurden diese Ergebnisse von vielen so ausgelegt, dass die Substitution mit Heroin i.v. zugunsten der oralen Methadonsubstitution aufgegeben werden sollte. Nach einer intensiven Diskussion begannen viele Kliniken einem mehr interventionistischen therapeutischen Ansatz zu folgen und weigerten sich, neuen Patienten injizierbare Drogen zu verschreiben [26]. 1994 erhielten in Großbritannien lediglich 1-2\% der geschätzten 75000-150000 Heroinkonsumenten rezeptierte injizierbare Drogen irgendeiner Art und nur ein geringer Teil von ihnen bekam injizierbares Heroin [25].

Die neuesten Erfahrungen mit der Verschreibung von anderen Opioiden als oralem Methadon kommen aus der Schweiz. 1994 wurde in der Schweiz eine groß angelegte Studie gestartet, in der die Behandlungsfolgen einer Verschreibung von injizierbarem und inhalierbarem Heroin, injizierbarem und oralem Morphin sowie injizierbarem und oralem Methadon untersucht wurden. Das Verschreibungsprogramm begann als umfassender wissenschaftlicher Versuch und wurde am 31. Dezember 1996 abgeschlossen. Die Auswertung zeigte sehr positive Ergebnisse für die 1146 Patienten, die Heroin i.v. erhalten hatten. Es gab außerdem eine bemerkenswerte öffent- 
liche Unterstützung für dieses Projekt, wie bei einem landesweiten Volksentscheid zu diesem Thema festgestellt werden konnte (das Projekt wurde insgesamt von $71 \%$ aller Wähler befürwortet und erzielte in jedem der 26 Kantone eine Mehrheit). Als Folge davon ist die ärztliche Verschreibung von Heroin heute in der Schweiz eine anerkannte Behandlungsmethode.

Nach den Berichten der Untersucher [24, 27, 28] und einer unabhängigen Verfahrensauswertung, die von der Weltgesundheitsorganisation [29] durchgeführt wurde, sind die Resultate der Schweizer Heroinstudie in der Tat als positiv zu werten. Die positiven Ergebnisse beinhalten hohe Haltequoten (89\% nach sechs Monaten und 69\% nach achtzehn Monaten), einschneidende Reduzierungen beim Gebrauch illegaler Drogen (Heroin und Kokain) und einen erheblichen Rückgang bei der Kriminalitätsrate der Teilnehmer. Die Studienteilnehmer verzeichneten außerdem erhebliche und stabile Verbesserungen in den Bereichen körperliche Gesundheit, psychisches Wohlbefinden, Wohn- und Arbeitssituation. Des Weiteren nahm die Zahl der Kontakte mit anderen Drogenkonsumenten und der Drogenszene allgemein erheblich ab. Ebenfalls wichtig ist, anzumerken, dass es hinsichtlich der öffentlichen Ordnung zu keinen Problemen kam und es keine Todesfälle gab, die auf den Versuch zurückzuführen wären. Schließlich zeigte auch die Kosten-Nutzen-Analyse beim Vergleich mit der üblichen Behandlung (vorwiegend Methadonsubstitution) einen wirtschaftlichen Gesamtnettogewinn von 32 US-Dollar (45 Schweizer Franken) pro Patient und pro Tag. Diese Ergebnisse basieren alle auf einem Eingruppen-vorher-nachher-Studiendesign. Im Rahmen dieses Ansatzes wurden die Baselinedaten mit katamnestisch erhobenen Zahlen von nahezu 800 Patienten verglichen, die 1994 in das Projekt aufgenommen wurden und wenigstens für die Dauer von 18 Monaten daran teilnahmen (69\%). Es bestand jedoch keine Möglichkeit, diese Vorher-nachher-Veränderungen mit ähnlichen Änderungen in einer randomisierten oder parallelisierten Kontrollgruppe, die wie üblich behandelt wurde, zu vergleichen. Erwähnenswert ist, dass die Schweizer Studie vier kleinere randomisierte Untersuchungen einschließt, wobei bei einigen ein Doppelblindverfahren angewendet wurde. Bei der Mehrzahl dieser Untersuchungen war es jedoch nicht beabsichtigt, die langfristigen Auswirkungen der Heroinverschreibung zu bewerten, sondern es ging eher darum, die kurzfristigen Auswirkungen, die in Verbindung mit den verschiedenen in der Studie verwendeten Substanzen (Heroin i.v., Methadon i.v., Morphin i.v.) auftraten, zu untersuchen, und zwar hinsichtlich ihres Einflusses auf die Haltequote, Compliance und Nebenwirkungen. Die einzige randomisierte Studie, welche die langfristigen Auswirkungen der Heroinverschreibung überprüft hat, war eine kleinere Studie, die in Genf durchgeführt wurde [30]. In dieser Studie wurden 51 therapieresistente Heroinabhängige randomisiert eingeteilt, um entweder eine Behandlung mit intravenösem Heroin und anderen psychosozialen Leistungen und Gesundheitsleistungen zu erhalten oder eine andere konventionelle Drogentherapie, die gewöhnlich in einer oralen Methadonsubstitution bestand. Nach sechs Monaten hatten nahezu alle Patienten, die legales Heroin bekamen, ihren illegalen Heroinkonsum eingestellt und ihre psychischen sowie sozialen Funktionen - gemessen anhand der Anzahl der Suizidversuche und der Kriminalitätsrate - hatten sich im Vergleich mit den Patienten der Kontrollgruppe deutlich stärker verbessert.
Es ist dabei zu berücksichtigen, dass diese neueren schweizerischen Studien nicht versuchen, die langfristigen Auswirkungen der alleinigen Heroinverschreibung, d.h. ohne obligatorische Beratung und andere psychosoziale Interventionen, $\mathrm{zu}$ untersuchen. Daher liefern sie keine Informationen über die Auswirkung der Heroinverschreibung per se, sondern eher über die Folgen einer Behandlung, die sich aus einem Bündel von pharmakologischen (Heroin) und psychosozialen Interventionen zusammensetzt. Es ist also möglich, dass die positiven Ergebnisse des schweizerischen Heroinsubstitutionsprogramms wenigstens zum Teil auf die zusätzlichen und häufig obligatorischen psychosozialen Interventionen zurückzuführen sind [30].

Abschließend ist anzumerken, dass der schweizerische Versuch mit inhalierbarem Heroin in Form von Heroinzigaretten (Surrogatzigaretten) aufgrund der niedrigen biologischen Verfügbarkeit (10-15\%) von Heroin aus diesen „Sugaretten“ nicht erfolgreich war. Die Forscher fanden heraus, dass 85$90 \%$ des Heroins bei dem Verbrennungsprozess zerstört wird und daher vom Patienten nicht aufgenommen werden kann.

Zusammengefasst scheint die ärztliche Verschreibung von injizierbarem Heroin bei chronischen, therapieresistenten Heroinabhängigen sowohl durchführbar als auch potenziell wirksam zu sein. Dies ist jedoch nur dann der Fall, wenn die Verschreibung ärztlich kontrolliert wird, keine Take-homeDosen erlaubt werden und psychosoziale Angebote zur Verfügung gestellt werden [u.a. 31]. Drei Fragen bleiben jedoch unbeantwortet. Die erste lautet: Worin besteht der spezielle Beitrag der Heroinverschreibung, wenn diese mit weiteren pharmakologischen und psychosozialen Maßnahmen kombiniert wird? Zweitens: Wie wirkt sich die Teilnahme an einer wissenschaftlichen Untersuchung auf die Vorher-nachher-Veränderungen bei einigen der wesentlichen Ergebnisvariablen aus? Und drittens: Könnte die Behandlung mit inhalierbarem Heroin positive Ergebnisse bringen, sollte es gelingen, die biologische Verfügbarkeit deutlich zu erhöhen? Besonders die letzte Frage ist für die Niederlande relevant, da sich dort nur 15-20\% der Heroinabhängigen die Droge intravenös zuführen und $80-85 \%$ das Heroin von der Folie rauchen.

Die gegenwärtige niederländische Studie über die Wirksamkeit der ärztlichen Verschreibung von Heroin versucht jede dieser Fragen zu beantworten. Dies soll im Rahmen einer randomisierten klinischen Studie erreicht werden, in der die Auswirkungen der Standardprogramme für orale Methadonsubstitution mit ähnlichen Programmen verglichen werden, in denen zusätzlich verschriebenes injizierbares oder inhalierbares Heroin verwendet wird, die Drogenabgabe in der Klinik stattfindet und keine Take-home-Dosen von Heroin erlaubt sind.

\section{Die Entscheidung, die niederländische Heroinverschreibungsstudie zu beginnen}

Wie bereits erwähnt, gibt es in den Niederlanden eine stabile, älter werdende Population von Heroinabhängigen mit langer Abhängigkeits- und Behandlungskarriere. Außerdem hat es sich gezeigt, dass ein erheblicher Anteil der niederländischen Heroinabhängigen therapieresistent ist, obwohl ein umfassendes und leicht zugängliches Behandlungssystem, das eine ganze Anzahl gut durchdachter Methadon-Substitutionspro- 
gramme anbietet, vorhanden ist. Diese Umstände sowie die positiven Ergebnisse der schweizerischen Erfahrungen veranlassten den damaligen Minister für Wohlfahrt, Gesundheit und kulturelle Angelegenheiten, den Gesundheitsrat der Niederlande um Rat zu fragen, welche Bedingungen zu erfüllen wären, damit die Heroinverschreibung als angemessenes klinisches Verfahren angesehen werden könnte.

In dem Antwortbericht auf diese Anfrage kam man zu dem Schluss, dass die ärztliche Verschreibung von Heroin an heroinabhängige Patienten positive Auswirkungen auf deren körperlichen und psychischen Zustand sowie auf das Suchtverhalten haben könnte. Die ärztliche Behandlung mit Heroin wurde als zweckdienlich angesehen, sofern durch fundierte medizinisch-wissenschaftliche Forschung belegt werden kann, dass dabei die Wirksamkeit der Therapie im Vergleich zu den mit einer solchen Behandlung verbundenen Schäden überwiegt. Um die notwendigen Daten zu bekommen, empfahl der Gesundheitsrat, in den Niederlanden eine Studie durchzuführen, in der solche schwer heroinabhängigen Patienten untersucht werden sollten, die erfolglos oder mit unzureichendem Erfolg an aktuell verfügbaren ärztlichen Interventionen teilgenommen hatten [32].

Im Anschluss daran entschied die niederländische Regierung in Übereinstimmung mit dem Parlament, die von dem Gesundheitsrat vorgeschlagene Studie vorzubereiten und durchzuführen. Im Dezember 1996 setzte dann der Minister für Gesundheit, Wohlfahrt und Sport das Zentrale Komitee für die Behandlung von Heroinabhängigen [33] ein und übertrug diesem die Aufgabe, über die geplanten Ziele und die negativen Auswirkungen der Heroinbehandlung zu berichten. Dieser Bericht sollte nach der Fertigstellung einer wissenschaftlichen Studie verfasst werden. Nach einer ausführlichen Diskussion entwickelte das Zentrale Komitee für die Behandlung von Heroinabhängigen (CCBH) Protokolle für die Untersuchung der Auswirkungen der ärztlichen Heroinverschreibung (entweder durch Injektion oder durch Inhalation) bei schwer kranken, therapieresistenten Heroinabhängigen. Die Protokolle wurden entwickelt in Übereinstimmung mit den Richtlinien für klinische Prüfung, dem niederländischen Recht und den allgemeinen medizinisch-ethischen Standards, welche die Verhaltensregeln für die medizinisch-wissenschaftliche Forschung festlegen. Außerdem wurden Protokolle für die Entwicklung und Testung einer stabilen und wirksamen inhalierbaren Form der Heroingabe entworfen. Diese Richtlinien wurden verschiedenen internationalen Experten vorgelegt, um deren Meinung einzuholen. Des Weiteren kam es zu einem umfangreichen Austausch von Ideen und Erfahrungen zwischen dem $\mathrm{CCBH}$ und verschiedenen Mitarbeitern des schweizerischen Projekts. Schließlich wurden umfangreiche Diskussionen mit dem Niederländischen Zentralkomitee für Medizinische Ethik geführt. Die Ergebnisse dieser und anderer Kontakte wurden in die endgültigen Protokolle aufgenommen.

Im August 1997 wurden dem Minister für Gesundheit, Wohlfahrt und Sport die endgültigen Protokolle vorgelegt. Das Studiendesign sah die Teilnahme von 750 Patienten vor und die Studie sollte in acht Behandlungseinheiten in verschiedenen niederländischen Städten durchgeführt werden. Im September 1997 genehmigte das niederländische Parlament eine dreimonatige Pilotperiode, während der insgesamt $185 \mathrm{~Pa}-$ tienten, von denen 50 Heroin erhalten, untersucht werden sollten. Sollte es zu keinen inakzeptablen medizinischen oder öffentlichen Problemen kommen, würde die Studie auf die volle Teilnehmerzahl von 750 Patienten ausgedehnt werden. Im November 1997 wurde festgelegt, dass die Pilotstudie in Amsterdam und Rotterdam durchgeführt werden soll. Im Januar 1998 bestätigte der International Narcotics Control Board (INCB) die für die Studie benötigte geschätzte Heroinmenge. Im Juli 1998 öffneten die ersten Behandlungseinheiten in Amsterdam und Rotterdam. Kürzlich öffneten Behandlungseinheiten in Den Haag, Groningen, Heerlen, Utrecht. Die endgültigen Ergebnisse der Studie werden im Jahr 2002 vorliegen.

\section{Die ärztliche Verschreibung von Heroin in den Niederlanden: eine randomisierte Studie}

\section{Die Ziele der Studie}

Das vorrangige Ziel der niederländischen Studie besteht in einer Beurteilung der nützlichen und schädlichen Auswirkungen der zwölfmonatigen Substitutionsbehandlung mit oralem Methadon und zusätzlich verschriebenem Heroin im Vergleich zur Standard-Substitutionsbehandlung mit nur oralem Methadon. Die Studienpopulation besteht aus chronischen therapieresistenten Heroinabhängigen, die gegenwärtig in Methadon-Substitutionsprogrammen aufgenommen sind. Für die Bewertung der Auswirkungen der ärztlichen Verschreibung von Heroin wird eine anhaltende Abstinenz nicht als primärer Ergebnisparameter herangezogen. Stattdessen werden von den Untersuchern Verbesserungen hinsichtlich des körperlichen und psychischen Zustands der Patienten, ihrer sozialen Integration und sozialen Funktionen sowie der Reduzierung ihres illegalen Drogenkonsums gemessen. Das Projekt beinhaltet verschiedene sekundäre Studienziele. Diese umfassen einen Vergleich der Auswirkungen des zusätzlich verschriebenen Heroins nach sechs und zwölf Monaten, die Auswirkungen des Behandlungsabbruchs mit zusätzlich verschriebenem Heroin nach sechs und zwölf Monaten, eine Beurteilung der Auswirkungen des zusätzlich verschriebenen Heroins auf das Wohlbefinden der Patienten und die Entwicklung von Hypothesen für zukünftige Behandlungsstrategien. Jedes dieser Ziele wird getrennt für die Verschreibung von injizierbarem und inhalierbarem Heroin beurteilt.

\section{Die Zielpopulation}

Nach dem Auskunftsbericht des Gesundheitsrates der Niederlande [32] sollte die Studie die Behandlung von schwer abhängigen Heroinkonsumenten untersuchen, die erfolglos oder mit unzureichendem Erfolg an aktuell zur Verfügung stehenden medizinischen Maßnahmen teilgenommen haben. Die niederländische Regierung fügte die Auflage hinzu, dass die Studie sich auf „ältere Patienten mit langer Suchtkarriere, deren psychosoziale Situation ohne Perspektive ist" beschränken sollte. In dem Studienprotokoll war diese Population operationalisiert definiert als Gruppe chronischer Heroinabhängiger, die wiederholt, jedoch ohne Erfolg, an Methadon-Substitutionsprogrammen teilgenommen haben. Die Entscheidung, sich bei der Studie auf diese Gruppe zu beschränken, basierte auf folgenden Überlegungen: Die Beschreibung der Zielgruppe trifft auf viele der Patienten in Methadon-Substitutionsprogrammen zu; die Probleme der Patienten in einer Methadonerhaltungstherapie sind in den 
Tab. 1 Aufnahme- und Ausschlusskriterien der niederländischen Heroinstudie
Aufnahmekriterien
1. therapieresistente Heroinabhängigkeit, die gekennzeichnet ist durch
a. anamnestische Heroinabhängigkeit > 5 Jahre;
b. mindestens $60 \mathrm{mg}$ (i.v.) oder $50 \mathrm{mg}$ (inhalieren) Methadon täglich
während eines ununterbrochenen Zeitraums von wenigstens
4 Wochen in den letzten 5 Jahren;
c. im letzten Jahr bestand regelmäßiger Kontakt mit einem
Methadonprogramm;
d. (nahezu) täglicher Konsum von illegalem Heroin;
e. schlechter körperlicher und/oder psychischer Gesundheitszustand und/oder schlechte soziale Funktionen
2. Heroin wird intravenös injiziert oder inhaliert

3. mindestens 25 Jahre alt

4. niederländischer Staatsbürger oder mit offiziellem Wohnsitz in den Niederlanden

5. seit mindestens 3 Jahren im Bereich des Behandlungszentrums als Bewohner gemeldet

6. ist einverstanden, während des Versuchszeitraums das Behandlungszentrum wenigstens dreimal wöchentlich aufzusuchen

7. schriftliche Einverständniserklärung
Ausschlusskriterien

1. Person erfüllt nicht alle Aufnahmekriterien

2. schwere medizinische, psychische oder psychosoziale Probleme, aufgrund derer eine Behandlung aus gesundheitlichen Gründen kontraindiziert ist

3. schwere medizinische, psychische oder psychosoziale Probleme, welche die Durchführung der Studie beeinträchtigen könnten

4. aggressives Verhalten in der Anamnese, das sich durch wiederholte Ausschlüsse aus Methadonprogrammen gezeigt hat

5. Schwangerschaft oder anhaltende Laktationsperiode

6. Personen, die nicht imstande oder willens sind, die Behandlungszentren für eine regelmäßige Beurteilung aufzusuchen

7. kurze Lebenserwartung

8. die Heroinabhängigkeit ist im Vergleich zu einer bestehenden Nicht-Opiat-Abhängigkeit von zweitrangiger Bedeutung

9. eine Periode freiwilliger Heroinabstinenz von mindestens zweimonatiger Dauer im vorangegangenen Jahr

10. Personen, die eine tägliche orale Methadondosis von mehr als $150 \mathrm{mg}$ benötigen

11. Personen, die eine tägliche Dosis zusätzlich verschriebenen Heroins von mehr als $1000 \mathrm{mg}$ benötigen

12. Personen, die gegenwärtig an einer anderen Studie über Entwöhnungsbehandlungen teilnehmen, oder Personen, die in den vergangenen sechs Monaten an einer solchen Studie teilgenommen haben
Niederlanden denen der Heroinkonsumenten, die gegenwärtig keinerlei Kontakt mit dem Behandlungssystem haben, sehr ähnlich [34]; die Registrierungssysteme, die mit den Methadonprogrammen verbunden sind, liefern einen klaren Stichprobenrahmen für die Auswahl der Studienteilnehmer; und durch die Kombination der Daten aus dem Methadonund dem Heroinregistrierungssystem kann das Verschreiben doppelter Dosen verhindert werden. Die Entscheidung, die Studiengruppe auf therapieresistente Abhängige zu begrenzen, die bereits an Methadonprogrammen teilgenommen haben, sollte dazu beitragen, eine höhere interne Validität sicherzustellen bei nur geringen Einschränkungen bezüglich der externen Validität. In Tab. 1 sind die Aufnahme- bzw. Ausschlusskriterien für die Zielpopulation dargelegt.

Das Aufnahmekriterium 1 (a-e) beschreibt den Schweregrad und die Chronizität der Probleme des Abhängigen, die sich ergaben, obwohl die Person in der Vergangenheit relativ hohe Dosen Methadon erhalten hat. Der im Kriterium 1a aufgeführte Cut-off-Punkt von fünf Jahren wurde aufgrund der systematischen Übersichtsarbeit von Cramer und Schippers [35] über den Verlauf und die Folgen der Heroinsucht gewählt. Dabei war herausgefunden worden, dass die bedeutendste Verlagerung vom Drogenkonsum und zur Abstinenz innerhalb der ersten fünf Jahre der Suchtkarriere erfolgt. Grob gesagt sind ungefähr 20\% der Population der problematischen Drogenkonsumenten nach fünf Jahren abstinent. Dieser Prozentsatz ändert sich nur unwesentlich in den folgenden fünf bis zehn Jahren. Bezüglich des Aufnahmekriteriums 1b ist es wichtig, zu beachten, dass es sich bei der Methadondosis von $80 \mathrm{mg}$ plus/minus $20 \mathrm{mg}$ pro Tag um die in den American State Methadone Maintenance Treatment Guidelines [12] empfohlene Effektivdosis handelt. Daher kann eine tägliche Dosis von $60 \mathrm{mg}$ als Mindestvoraussetzung für eine wirksame Methadontherapie angesehen werden. Folglich wurde die Mindestdosis von $60 \mathrm{mg}$, die über wenigstens vier aufeinander folgende Wochen gegeben wurde, als ein Kriterium für die Entscheidung herangezogen, ob ein Patient ein dem aktuellen Stand entsprechendes Methadon-Substitutionsprogramm erfolglos absolviert hat.

Hinsichtlich der Ausschlusskriterien ist es wichtig, festzuhalten, dass diese bewusst, ohne explizit operationalisiert zu werden, formuliert wurden, da dadurch nur jene Patienten ausgeschlossen werden sollten, bei denen es sehr wahrscheinlich ist, dass sie die Durchführung der Studie gefährden würden. Es wird erwartet, dass nicht mehr als 2-3\% der angeworbenen Patienten von der Teilnahme an der Studie ausge- 


\begin{tabular}{|c|c|c|c|c|c|}
\hline $\begin{array}{l}\text { Verabrei- } \\
\text { chungsart }\end{array}$ & Gruppe & $\begin{array}{l}\text { Phase I } \\
4-8 \text { Wochen }\end{array}$ & $\begin{array}{l}\text { Phase lla } \\
6 \text { Monate }\end{array}$ & $\begin{array}{l}\text { Phase llb } \\
6 \text { Monate }\end{array}$ & $\begin{array}{l}\text { Phase III } \\
6 \text { Monate }\end{array}$ \\
\hline \multirow[t]{3}{*}{ Inhalieren } & A & $\mathrm{M}$ & $M$ & M & $M+H(i h)$ \\
\hline & B & $\mathrm{M}$ & $M+H(i h)$ & $M+H(i h)$ & $\begin{array}{l}\text { maximal geeig- } \\
\text { nete Betreuung }\end{array}$ \\
\hline & $\mathrm{C}$ & $\mathrm{M}$ & $\mathrm{M}$ & $M+H(i h)$ & $\begin{array}{l}\text { maximal geeig- } \\
\text { nete Betreuung }\end{array}$ \\
\hline \multirow[t]{3}{*}{ Injizieren } & A & $\mathrm{M}$ & M & $M$ & $\mathrm{M}+\mathrm{H}$ (iv) \\
\hline & B & $\mathrm{M}$ & $\mathrm{M}+\mathrm{H}$ (iv) & $M+H(i v)$ & $\begin{array}{l}\text { maximal geeig- } \\
\text { nete Betreuung }\end{array}$ \\
\hline & $C$ & $M$ & $M$ & $M+H(i v)$ & $\begin{array}{l}\text { maximal geeig- } \\
\text { nete Betreuung }\end{array}$ \\
\hline
\end{tabular}

Tab. 2 Studiendesign der niederländischen Heroinstudie

$M=$ orales Methadon; $H$ (ih) = inhalierbares Heroin; $H$ (iv) = injizierbares Heroin

schlossen werden. Aus diesem Grund stellen diese Kriterien keine ernsthafte Gefahr für die externe Validität der Studie dar. Da bei den Patienten, die das Ausschlusskriterium 9 erfüllen, weiterhin die Möglichkeit besteht, dass sie mit ihren Abstinenzbemühungen erfolgreich sind, werden sie von der Studie ausgeschlossen.

\section{Studiendesign}

Wie bereits an anderer Stelle erwähnt, wurde die Studie für 750 Teilnehmer entworfen, die in acht verschiedenen Behandlungseinheiten in sechs niederländischen Städten betreut werden sollen. Nach einer intensiven Diskussion hat das Zentrale Komitee für die Behandlung von Heroinabhängigen $(\mathrm{CCBH})$ entschieden, dass eine herkömmliche randomisierte klinische Studie einer so genannten Prä-Randomisierungsstudie oder Zelen-Design vorzuziehen sei [33,36,37]. Innerhalb dieser randomisierten Studie (Phase II) sind drei unterschiedliche Behandlungsformen zu unterscheiden: 1. Patienten der Gruppe A erhalten während der ersten zwölf Monate der Studie orales Methadon kombiniert mit einer Reihe von psychosozialen Standardmaßnahmen; 2. den Patienten der Gruppe B wird während der ersten zwölf Monate der Studie orales Methadon und Heroin in Kombination mit den gleichen psychosozialen Standardmaßnahmen angeboten; 3. die Patienten der Gruppe C erhalten in den ersten sechs Monaten der Studie orales Methadon kombiniert mit den psychosozialen Standardmaßnahmen und in den zweiten sechs Monaten der Studie erhalten sie eine Kombination aus Methadon und zusätzlich verschriebenem Heroin in Verbindung mit den gleichen psychosozialen Standardmaßnahmen (siehe Tab.2). Aufgrund der geringeren Patientenzahl, die unter injizierenden Heroinabhängigen rekrutiert werden konnte - etwa 80-85\% der niederländischen Heroinabhängigen inhalieren - wurde beim Injektions-Protokoll auf die Gruppe C verzichtet. Damit beläuft sich die Gesamtzahl aller Untersuchungsteilnehmer auf 625 Patienten, 375 im Inhalationsprotokoll und 250 im InjektionsProtokoll. Die Patienten werden erst nach Ablauf einer vierbis achtwöchigen Qualifikationsperiode (Phase I) einer dieser Behandlungsoptionen randomisiert zugeteilt.

Die Hauptauswertungen der Ergebnisse finden nach sechs (Phase IIa) und zwölf (Phase IIb) Monaten der Behandlung statt, d.h. sechs und zwölf Monate nach erfolgter Randomisierung. Im Anschluss an Phase IIb beginnt für alle Teilnehmer eine Follow-up-Periode von sechs Monaten (Phase III). Für die
Patienten der Gruppe A besteht nun die Möglichkeit, für den Zeitraum von sechs Monaten die Behandlung mit zusätzlich verschriebenem Heroin fortzusetzen. Die Heroinverschreibung in den Gruppen B und C wird am Ende der Phase IIb eingestellt und durch das Angebot ersetzt, wieder die MethadonSubstitutionsbehandlung aufzunehmen oder eine andere Standard-Suchttherapie zu beginnen. Dies gilt sowohl für Responder als auch für Non-Responder der Versuchsbehandlung mit Heroin. Für die Gruppe der Non-Responder in Phase II ist die Beendigung der Heroinverschreibung endgültig. Ebenso erhalten auch diejenigen Patienten, die positiv auf die Versuchsbehandlung reagiert haben und deren Zustand sich nach Beendigung der Heroinverschreibung nicht verschlechtert, kein Heroin mehr. Die Heroinverschreibung kann bei den Respondern auf die Versuchsbehandlung, die in den ersten zwei Monaten nach Einstellung der experimentellen Behandlung eine erhebliche Verschlechterung ( $>20 \%$ bezogen auf den Schweregrad, festgehalten in den Ausgangsdaten) in ihren Funktionen zeigen, wieder aufgenommen werden. In diesem Fall erfolgt die Verschreibung von Heroin auf ärztliche Empfehlung und auf streng individueller Basis.

\section{Behandlungen}

Bei den in der Studie verschriebenen Drogen handelt es sich um Heroin (Hydrochlorid oder Base) und Methadon (Hydrochlorid). Wie zuvor erwähnt, wird den Patienten in den Versuchsgruppen sowohl Methadon als auch Heroin in Kombination mit einer Reihe von psychosozialen Standardmaßnahmen angeboten, während die Patienten der Kontrollgruppe lediglich Methadon und ebenfalls die gleichen psychosozialen Leistungen erhalten. Methadon wurde deshalb als Kontrollsubstanz gewählt, weil es das am besten untersuchte Substitutionsmedikament darstellt, das derzeit verfügbar ist. Heroin wurde als Versuchssubstanz gewählt, weil es im Vergleich mit den meisten anderen Opioiden bessere Ergebnisse bezüglich der Attraktivität für die Patienten, der Haltequote, der Compliance und der Anzahl der problematischen Nebenwirkungen erbrachte [28]. Der Grund für das Verwenden einer Kombination von Methadon und (zusätzlich verschriebenem) Heroin in den Versuchsgruppenliegt darin, dass Methadon, das eine lange Halbwertszeit hat, das Auftauchen von Entzugserscheinungen verhindern kann, wenn die Einnahme von Heroin nicht möglich oder wünschenswert ist. Sowohl Methadon als auch Heroin sind für alle Teilnehmer der Studie kostenlos. Methadon wird als wirksames Medikament ange- 
sehen und daher in den Niederlanden von den Krankenkassen voll bezahlt. Heroin hingegen wird als experimentelles Medikament ohne erwiesene Wirkung behandelt und kann deshalb dem Patienten nicht in Rechnung gestellt werden. In der Injektionsgruppe wird das Heroin als 20\%ige Diacethylmorphin-Hydrochlorid-Lösung verwendet, während es in der Inhalationsgruppe als Pulver verwendet wird, das aus einer Diacethylmorphinbase und Koffein besteht. Letzterer Bestandteil wird hinzugefügt, um das Verdampfen von Diacethylmorphin zu erleichtern, was zu einer optimalen Bioverfügbarkeit des Heroin führt [38]. Die Dosen werden individuell titriert mit maximal $400 \mathrm{mg}$ reinem Heroin pro Verabreichung und maximal $1000 \mathrm{mg}$ reinem Heroin pro Tag in einem Behandlungsregime mit maximal drei Verabreichungen täglich und einem Behandlungssetting, das während sieben Tagen in der Woche Behandlungen ermöglicht. Im Allgemeinen verabreichen sich die Patienten das Heroin selbst, entweder durch eine Injektion oder das Rauchen von der Folie. Den Patienten, die der Injektionsgruppe randomisiert zugeteilt worden waren, ist es gestattet, auf das Inhalieren von Heroin umzusteigen. Den Patienten, die der Inhalationsgruppe zugeordnet sind, ist es jedoch nicht erlaubt, sich stattdessen das Heroin zu injizieren. Take-home-Dosen von Heroin sind nicht gestattet und es werden keine illegalen Drogen an die Studienteilnehmer verschrieben.

Den Patienten in den Versuchsgruppen und denen in der Kontrollgruppe werden die gleichen psychosozialen Maßnahmen angeboten, einschließlich individueller Beratung, Gruppenberatung, Unterbringung und Schuldnerberatung, Teilnahme an Arbeitsprojekten sowie medizinische und psychiatrische $\mathrm{Be}$ handlung. Diese zusätzlichen Behandlungsangebote stehen allen Patienten, die in den Niederlanden an bestehenden Methadon-Substitutionsprogrammen teilnehmen, zur Verfügung, wenngleich sie sich in den verschiedenen Städten in Umfang und Kombinationsmöglichkeiten unterscheiden [39]. Aufgrund der Unterschiede zwischen den Städten hinsichtlich der Verfügbarkeit dieser zusätzlichen Behandlungsangebote entschloss man sich, die randomisierten Zuteilungen je nach Stadt zu schichten.

\section{Ergebnis- und Erfolgsparameter}

Es wird in der vorgesehenen Studie erwartet, dass eine Stabilisierung oder Einstellung des illegalen Drogenkonsums mit einer Verbesserung des körperlichen und/oder psychischen Gesundheitszustands sowie der sozialen Funktionen und sozialen Integration einhergeht. Im Hinblick auf die primäre Ergebnisvariable muss jedoch darauf geachtet werden, wie diese verschiedenen Ergebnisbereiche zusammenhängen. Es sind dabei zwei Ansätze berücksichtigt worden. Der erste beruht auf der Ansicht, dass der wichtigste und wohl auch am schwierigsten zu erzielende Nutzen der Behandlung bei dieser spezifischen Population in dem Erreichen sozialer Integration und Rehabilitation besteht. Frühere Untersuchungen deuten darauf hin, dass einer verbesserten sozialen Integration und Rehabilitation eine initiale Reduzierung oder Stabilisierung des illegalen Drogenkonsums und der illegalen Aktivitäten folgen. Dies trifft ebenso für Verbesserungen des körperlichen und psychischen Gesundheitszustands zu. Bei diesem Ansatz bilden die soziale Integration und Rehabilitation die primäre Ergebnisvariable. Verbesserungen beim illegalen Drogenkonsum und im Gesundheitszustand werden als
Vorboten einer verbesserten sozialen Integration betrachtet. Veränderungen beim Gesundheitszustand oder dem illegalen Drogengebrauch werden daher als sekundäre Ergebnis- oder Prozessvariable angesehen. Folglich sollte die Teststärke der Studie auf entsprechenden Zielen und realistischen Erwartungen hinsichtlich der Auswirkungen auf die soziale Rehabilitation beruhen.

Beim zweiten Ansatz werden keine Annahmen bezüglich der Hierarchie oder Reihenfolge der verschiedenen Ergebnisbereiche vorgenommen. Zur Erfassung des primären Wirkungsgrades wird ein zusammengesetzter Index Score erstellt, der sich gleichzeitig auf Verbesserungen beim illegalen Drogenkonsum, dem körperlichen und psychischen Gesundheitszustand sowie bei den sozialen Funktionen bezieht, um einen Behandlungseffekt zu zeigen. Infolgedessen sollte bei diesem Ansatz die Teststärkenanalyse auf dem zusammengesetzten Index Score basieren. Außerdem gilt es, die Frage zu klären, ob die primäre Ergebnisvariable dimensional oder kategorial sein sollte. Erstere wird oft anhand der Unterschiede bei den durchschnittlichen Besserungsraten der Versuchs- und der Kontrollgruppe(n) dargestellt, während Letztere gewöhnlich mithilfe der Unterschiede bei der Response-Rate der Versuchs- und der Kontrollgruppe(n) ausgedrückt wird. In beiden Fällen sollten jedoch vor dem Beginn der Studie der Unterschied, der als klinisch relevant anzusehen ist, sowie die statistische Analyse festgelegt werden. In einer Konsenserklärung des European College of Neuropsycho-Pharmacology [40] wurde entschieden, dass „(...) ein statistisch signifikanter Unterschied zwischen einer Medikamenten- und einer Plazebobehandlung bezogen auf den Prozentsatz der Responder, definiert als ein vorher festgelegter Grad der Reduzierung auf einer Pivottabelle, wohl die objektivste Information über die klinische Bedeutsamkeit darstellt, die zur Zeit verfügbar ist.“ Nach einer langen Diskussion legte das Zentrale Komitee für die Behandlung von Drogenabhängigen ( $\mathrm{CCBH})$ fest, dass der prozentuelle Unterschied bei den Respondern zwischen der Versuchsgruppe und der Kontrollgruppe mindestens 20\% betragen sollte. Ein Responder wurde definiert als Patient, der folgende Kriterien erfüllt: a) verbessert ihren oder seinen medizinischen Zustand um mindestens 40\% UND/ODER b) verbessert ihre oder seine soziale Funktionen um mindestens 40\% UND c) zeigt verglichen mit den Ausgangsdaten keine Verschlechterung von $40 \%$ oder mehr bei den medizinischen oder sozialen Funktionen UND d) steigert den illegalen Drogenkonsum um nicht mehr als $20 \%$.

\section{Erste Erfahrungen}

Seit dem Beginn der Rekrutierung im Juli 1998 wurden 370 Patienten für das Injektionsprotokoll beziehungsweise 160 für das Inhalationsprotokoll gewonnen. Von diesen $530 \mathrm{~Pa}-$ tienten sind zur Zeit 180 in Phase III der Studie. Während der Rekrutierung und Behandlung dieser Gruppe sind keine schwerwiegenden medizinischen Negativeffekte bezogen auf die Heroinverschreibung zu beobachten gewesen, und es gab auch keine ernsten Störungen der öffentlichen Ordnung. Die Randomisierung verursachte keine schwerwiegenden Probleme, und das Follow-Up nach 12 Monaten erreichte $96 \%$ der Patienten. Es kam außerdem zu keinen größeren Schwierigkeiten bei der Absetzung des Heroins in den Gruppen B und $C$ am Ende der Phase II. Zur Zeit sind noch keine Daten verfügbar bezüglich der Haltequoten und dem Anteil der po- 
sitiv reagierenden Patienten in den verschiedenen Behandlungsgruppen. Diese Ergebnisse werden voraussichtlich im Frühjahr 2002 vorliegen.

\section{Diskussion}

In diesem Artikel sind der historische Hintergrund und die klinischen Gründe für die niederländische Studie über die ärztliche Verschreibung von Heroin an chronische, therapieresistente Methadonpatienten dargelegt worden. Hervorzuheben ist, dass die ärztliche Verschreibung von Heroin nicht als Therapie erster Wahl für Patienten mit einer Heroinsucht anzusehen ist und dass es sich bei der ärztlichen Verschreibung von Heroin nicht um einen Ersatz für gegenwärtig verfügbare und gut eingeführte Behandlungen wie der oralen Methadonsubstitution handelt. Vielmehr stellt die Heroinsubstitution eine zusätzliche, letzte Behandlungsoption dar als Bestandteil eines leicht zugänglichen, umfassenden Behandlungssystems. Die ärztliche Heroinverschreibung sowie die Studien über seine Wirksamkeit sollten niemals auf Kosten bereits bestehender wirksamer Therapien angeboten werden. Diese Therapieform könnte sich jedoch für einige Patienten, die auf andere Behandlungen nicht ansprechen, als nützlich erweisen.

Die Autoren sind der Auffassung, dass die Schweizer Studie über ärztliche Heroinverschreibung gezeigt hat, dass eine solche Behandlung durchführbar ist, obgleich viele wichtige Fragen noch unbeantwortet sind. Die bedeutendsten Fragen betreffen den Wirkungsmechanismus bei der Heroinverschreibung sowie die Durchführbarkeit und Wirksamkeit der ärztlichen Verschreibung von inhalierbarem Heroin. Es ist anzumerken, dass es sich bei der randomisierten klinischen Studie nicht um das einzige, möglicherweise nicht einmal um das am besten geeignete Design handelt, um ein differenziertes Verständnis für die psychologischen und sozialen Veränderungen zu fördern, zu denen es beim Einzelnen und zwischen Heroinabhängigen in den Drogen- und kriminellen Subkulturen kommt. Aus diesem Grund wird die klinische Studie um weitere qualitative Untersuchungen ergänzt, in denen die wechselnde Funktion von Heroin unter den Patienten der Versuchsgruppe, damit in Zusammenhang stehende Veränderungen in deren Lebensstil ebenso wie die Auswirkungen der neuen Therapie auf die Drogenszene insgesamt erforscht werden.

Abhängig vom Verlauf und den Ergebnissen der niederländischen Studie ergeben sich unterschiedliche Szenarien für die Zukunft. Häufiges Auftreten unüberwindlicher öffentlicher Probleme, schwere negative medizinische Zwischenfälle oder fehlende Belege für eine klinisch relevante positive Wirksamkeit würden fast unweigerlich zu einer Einstellung dieser Therapieformen in den Niederlanden führen. Im Gegensatz dazu könnten sich durch positive Ergebnisse eine Anzahl neuer Fragen ergeben. Zunächst wäre zu klären, ob ausreichend Daten zur Verfügung stehen, um die ärztliche Heroinverschreibung als routinemäßige Behandlungsoption im Rahmen des umfassenden Behandlungssystems durchzuführen. Andere Fragen betreffen die am besten geeigneten Zielpopulationen für diese Therapieform. Sollte sie auf die Patienten beschränkt bleiben, welche die in der ursprünglichen Studie formulierten Aufnahmekriterien erfüllen, oder sollten auch andere Heroinabhängige für eine Teilnahme an der ärztlichen
Heroinverschreibung berücksichtigt werden? Und wenn ja, sollte dies dann ohne förmliche wissenschaftliche Auswertung geschehen oder sollten die gleichen strengen Untersuchungen wie in der beschriebenen randomisierten Studie vorgenommen werden? Diese und weitere Fragen zeigen, dass die niederländische Studie über die Verschreibung von Heroin erst der Beginn einer interessanten neuen Ära ist, die innovative Behandlungsoptionen bringen wird und in der neue Studien durchgeführt werden. Eines Tages werden diese neuen Therapien in das bestehende verfügbare Behandlungssystem aufgenommen und integriert worden sein. Weitere interessante Fragen zur Kosteneffizienz, dem Patient-BehandlungsMatching und der politischen Akzeptanz bestimmter wirksamer Interventionen sind noch ungeklärt. Kreative Diskussionen sind zu führen und innovative Lösungen zu finden, um die Möglichkeiten für eine Verbesserung der Situation aller Abhängiger und der Gesellschaft insgesamt ausnutzen zu können.

\section{Literatur}

${ }^{1}$ Korf DJ, Nabben T. Dutch Treat: Formal Control and Illicit Drug Use in the Netherlands. Thesis Publishers, Amsterdam: 1995

2 Schreuder RF, Broex VMF. Verkenning Drugsbeleid in Nederland: Feiten Opinies en Scenarios. Zoetermeer, STG, 1998

${ }^{3}$ Hoekstra MJ, Derks J. Verslaving, verslavingszorg en verslavingsbeleid in Nederland: een overzicht. In: Derks J, Hoekstra M (Hrsg). Verslavingszorg, een Vak Apart, Utrecht: NcGv, 1991: 57-92

4 van Brussel GHA, Buster MCA, van der Woude DH. Dovend Vuur: Jaarbericht Drugsafdeling 1994-1995. G G\&GD, Amsterdam: 1996

${ }^{5}$ Buster M, Reurs H. Methadon Verstrekking in Amsterdam 1997. Centrale Methadon Registratie, Amsterdam: GG\&GD, 1997

${ }^{6}$ Toet J. Tabellenboek RODIS, de Verslavingszorg in Rotterdam van 1991 to 1995. GGD, Rotterdam: 1996

7 van Galgendonk A, de Zwart W, van der Stel J, Donker M. De Nederlandse Verslavingszorg: Overzicht van de Kennis over Aanbod, Vvraag en Effect. Trimbos Instituut, Utrecht: 1997

8 Bieleman B, Snippe J, de Bie E. Drugs Binnen de Grenzen, Harddrugs en Criminaliteit in Nederland, Schatlingen van de Omvang. Intraval, Groningen/Rotterdam: 1995

${ }^{9}$ Office of National Drug Control Policy (ONDCP). National Drug Control Strategie 1998. (www.whitehousedrugpolicy.gov), 1998

10 Driessen FMHM. Methadonverstrekking in Nederland. Bureau Driessen, Utrecht: 1990

11 Jongerius J, Hull H, Derks J. Hoe Scoort de Verslavingszorg. NcGv, Utrecht: 1994; 9

12 Parrino MW. State Methadone Maintenance Treatment Guidelines. U.S. Department of Health and Human Services, Center for Substance Abuse Treatment, Rockville Maryland: 1992

${ }^{13}$ DAunno $T$, Vaughn TE. Variations in methadone treatment practices: Results from a national study. JAILM 1992; 267: 253257

${ }^{14}$ Korf DJ, Lettink D, Bouma H. Methadondosering in Nederland, een Exploratief Onderzoek onder Verstrekkers en Clienten Naar Hoge en Lage Doseringen Methadon. Amsterdams Bureau voor Onderzock en Statistiek, Amsterdam: 1998

${ }^{15}$ van Laar MW, van Ooyen-Houben M M, Spruit IP Netherlands Institute for Alcohol and Drugs. Fact sheet on Hard Drug Policy: Opiates. Netherlands Institute for Alcohol and Drugs, Utrecht: 1995

${ }^{16}$ de Zwart WM, van Wamel AL. Over Gebruik en Zorg in Cijfers. Jaarboek Verslaving 1997, Houten: Bohn Stafleu van Loghum, 1998 
${ }^{17}$ Havas A. Ervaring met de verstrekking van inspuitbare opiaten Maandblad Geestelijke Volksgezondheid 1983; 38: 1160-1167

18 Derks JTM. The dispensing of injectable morphine in Amsterdam: Experiences, results and implications for the Swiss project for the medical prescription of narcotics. In: Rhis-Middel M (Hrsg). The Medical Prescription of Narcotics - Scientific Foundations and Practical Experiences, Seattle: Hogrefe \& Huber, 1997: $167-180$

19 van Brussel GHA. The morphine distribution program in Amsterdam: Practical experiences. In: Rihs-Middel M (Hrsg). The Medical Prescription of Narcotics - Scientific Foundations and Practical Experiences, Seattle: Hogrefe \& Huber, 1997: 160-166

${ }^{20}$ Derks JTM. De verstrekking van injecteerbare opiaten: Doelstelling van he morfine-verstrekkingsexperiment. Tijdschrift voor Sociaile Gezondheidszorg 1984; 12: 496-498

${ }^{21}$ Derks JTM. Het Amsterdarnse Morfine-verstrekkingsexperiment. Doctoral dissertation, Utrecht: Rijksuniversiteit Utrecht, 1990

22 van Brussel GHA. Evaluatieverslag Palfium Behandeling voor Langdurig Heroine verslaafden. GG\&GD, Amsterdam: 1997

${ }^{23}$ Hartnoll RL, Mitcheson MC Battersby A. Evaluation of heroin maintenance in a controlled trial. Archives of General Psychiatry 1980; 37: 877-884

24 Uchtenhagen AF, Gutzwiller, Dobler-Mikola A, Blätter R. Program for a Medical Prescription of Narcotics: Interim Report of the Research Respresentatives. Zürich: Institut für Suchtforschung in Verbindung mit der Universität, Zürich: 1996

25 Strang J, Ruben S, Farrell M, Gossop M. Prescribing heroin and other injectabie drugs. In: Strang J, Gossop M (Hrsg). Heroin Addiction and Drug Policy: The British System, Oxford: Oxford University Press, 1994: 192-206

${ }^{26}$ Mitcheson M. Drug clinics in the 1970s. Strang J, Gossop M (Hrsg). Heroin Addiction and Drug Policy: 77, British System, Oxford: Oxford University Press, 1994: 178-191

27 Uchtenhagen AF, Dobler-Mikola A, Gutzwiller. Versuche für eine ärtzliche Verschreibung von Betäubungsmitteln: Zweiter Zwischenbericht der Forschungsbeauftragten. Institut für Suchtforschung in Verbindung mit der Universität Zürich, Zürich: 1996

28 Uchtenhagen AF, Gutzwiller, Dobler-Mikola A. Versuche für eine ärztliche Verschreibung von Betäubungsmitteln: Abschlussbericht der Forschungsbeauftragten. Institute für Suchtforschung in Verbindung mit der Universität Zürich, Zürich: 1997

${ }^{29}$ Report of the External Panel on the Evaluation of the Swiss Scientific Studies of Medically Prescribed Narcotics to Drug Addicts. World Health Organization, Geneva: 1999

30 Perneger TV, Giner F, del Rio M, Mino A. Randomized trial of heroin maintenance programme for addicts who fail in conventional drug treatments. British Medical Journal 1998; 317: 13-18

31 Wodak A. Prescribing heroin: Nothing else to fear but fear itsef? Illicit drug policy based on punitive measures has failed, and it is time to seek a healthcare approach. The Medical Journal ofAustralia 1998; 168: 590-591

32 Health Council of the Netherlands. The Prescription of Heroin to Heroin Addicts. The Hague: Health Council of the Netherlands, 1995

${ }^{33}$ Central Committee on the Treatment of Heroin Addicts. Investigating The Medical Prescription of Heroin. CCBH, Utrecht: 1997

34 Eland-Goossensen MA. Opiate Addicts In and Outside of Treatment: Different Populations? Erasmus Universiteit Rotterdam, Rotterdam: 1997

35 Cramer EASM, Schippers GM. Zelfcontrole en Ontwenning van Harddrugs. University of Nijmegen: Research Group on Addictive Behaviors, Nijmegen: 1994

${ }^{36}$ Zelen M. A new design for randomized controlled trials. New England Journal Of Medicine 1979; 300: 1242-1245

37 Zelen M. Randomized consentdesigns forclinical trials: An update. Statistics in Medicine 1990; 9: 645-646
${ }^{38}$ Huizer H. Analytical Studies on Illicit Heroin. Academic Dissertation, The Hague: Netherlands Ministry of Justice, 1988

39 van der Lelij B, Driessen FMHM. Psychosociale Hulpverlening voor de Doelgroep van het Heroineverstrekkings-experiment. Bureau Driessen, Utrecht: 1998

${ }^{40}$ European College of Neuropsychopharinacology. Methadonclienten in Nederland. Bureau Driessen, Utrecht: 1992

Wim van den Brink

Centrale Commissie Behandeling Heroinverslaafden (CCBH)

Stratenum-5e Verdieping

Universiteitsweg 100

3584 CG Utrecht

Niederlande

E-mail: CCBH@MED.UU.NL or W.VANDENBRINK@AMC.UVA.NL 